\title{
OBJECT SHAPE MORPHING WITH INTERMEDIATE REFLECTANCE PROPERTIES
}

\author{
YOICHI SATO* \\ Department of Electrical Engineering and Electronics \\ Institute of Industrial Science, The University of Tokyo \\ 7-22-1 Roppongi, Minato-ku, Tokyo 106, Japan \\ IMARI SATO \\ Department of Electrical Engineering and Electronics \\ Institute of Industrial Science, The University of Tokyo \\ 7-22-1 Roppongi, Minato-ku, Tokyo 106, Japan \\ and

\section{KATSUSHI IKEUCHI} \\ Department of Electrical Engineering and Electronics \\ Institute of Industrial Science, The University of Tokyo \\ 7-22-1 Roppongi, Minato-ku, Tokyo 106, Japan
}

Received (received date)

Revised (revised date)

Communicated by (Name of Editor)

\begin{abstract}
Image morphing techniques can create a smooth transition between two images. However, one of the main weakness of the image morphing technique is that intermediate images in the transition often have physically incorrect shading such as highlights and shadows. Moreover, we cannot alter viewing and lighting conditions when creating the intermediate images. That is because those images are obtained by simply interpolating pixel intensities of the two 2D images without knowledge of 3D object shape and reflectance properties. In this context, 3D shape morphing techniques have a definite advantage in that arbitrary viewing and illumination conditions can be used for creating new images. Unfortunately, previous 3D morphing techniques do not account for object surface reflectance properties or reflection models when generating intermediate images. This often results in undesired shading artifacts. In this paper, we consider a new approach for 3D shape and reflectance morphing of two real 3D objects. Our morphing method consists of two components: shape and reflectance property measurement, and smooth interpolation of those measured properties. The measured shape and reflectance parameters are used to compute intermediate shape and reflectance parameters. Finally, the computed shape and reflectance parameters are used to render intermediate images which represent a smooth transition between the two objects.
\end{abstract}

Keywords: Computer Vision, Color Image Analysis, Reflectance Measurement, Shape Morphing

\section{Introduction}

Recently, image morphing techniques for creating a smooth transition between two

*Contact the author at ysato@iis.u-tokyo.ac.jp. 
images have been extensively studied. These techniques are based on interpolation of coordinates (and colors) of image pixels in two images. Surprisingly convincing image transitions can be created when the interpolation is designed carefully [1] and [11].

However, one of the fundamental weaknesses of the image morphing technique is that intermediate images often have physically incorrect shading such as highlights and shadows. Moreover, we cannot create intermediate images under different viewing and lighting conditions. This is because those images are created by simply interpolating pixel intensities of the two 2D images without knowledge of viewing and illumination conditions and $3 \mathrm{D}$ object shapes. In this context, 3D shape morphing techniques have a definite advantage in that arbitrary viewing and illumination conditions can be used for creating new images [2], [5], and [6]. Unfortunately, those $3 \mathrm{D}$ morphing techniques do not account for object surface reflectance properties or reflection models for generating new images. This often results in undesired shading artifacts (e.g., shadows and highlights fail to match shape changes occurring in the morph).

Recently, techniques to measure object shape and reflectance properties by using both range images and intensity images have been studied. Ikeuchi and Sato [3] originally developed a method to measure object shapes and reflection function parameters from a range image and black and white intensity image pair. The surface shape was first recovered from the range image, and then the surface normals of the recovered object surface and the intensity image were used for reflectance parameter estimation. The main drawback of this method is that it assumes uniform reflectance properties over the object surface. Kay and Caelli [4] introduced another method to use a range image and 4 or 8 intensity images taken under different illumination conditions. By increasing the number of intensity images, they estimated reflection function parameters locally for each image pixel. Unlike the algorithm proposed by Sato and Ikeuchi, the method can handle object surfaces with varying reflectance properties. However, it is reported that parameter estimation can be unstable especially when the specular reflection component is not observed strongly. Sato and Ikeuchi [10] proposed another approach for measuring object shape and reflectance properties from a sequence of range and color images. This method differs from previous ones in that reflectance parameters are estimated based on results of reflection component separation. This makes parameter estimation more reliable than previously reported methods.

In this paper, we consider a new approach for $3 \mathrm{D}$ shape and reflectance morphing of two real 3D objects. Our morphing method consists of two components: shape and reflectance property measurement, and smooth interpolation of the measured properties. For measuring shape and reflectance parameters, the method proposed by Sato and Ikeuchi [10] is used in our analysis. First, a range image and a color image sequence are obtained for each of the two objects while the objects are rotated. Then, for each range image pixel, the sequence of observed colors of a surface point corresponding to the range image pixel is determined. The observed 
color sequence is separated into the diffuse and specular reflection components by the algorithm used originally by Sato and Ikeuchi [9]. Subsequently, parameters of a reflection function used in our analysis are estimated reliably for the separated reflection components. The reflection model used here is a linear combination of the Lambertian model and a simplified Torrance-Sparrow model [13]. The estimated reflectance parameters of each surface point are combined with its measured $(X, Y, Z)$ location, and then stored in a new $2 \mathrm{D}$ image. Therefore, each pixel of the $2 \mathrm{D}$ image has reflectance parameters as well as a $(X, Y, Z)$ location. The 2D image containing the $(X, Y, Z)$ location and reflectance parameters is referred to as an object image in this paper. After object images are created for the two objects, the two object images are used to create a new intermediate object image by interpolating the shape and reflectance properties of the two object images. Pixelwise correspondences between the two object images used for the interpolation is established from correspondence of manually drawn line pairs in the two object images [1]. Finally, the newly created intermediate object image is used to render new images of the object under arbitrary viewing and illumination conditions.

The paper is organized as follows. Section 2.1 and Section 2.2 describe our image acquisition system and determination of observed color sequences for each rangeimage pixel. Section 2.3 introduces the reflection model used in our experiment. The algorithm for separating the diffuse and specular reflection components is explained in Section 2.4, and reflectance parameter estimation is discussed in Section 2.5. Section 3 explains how to establish pixelwise correspondences between two object images based on manually drawn line pairs. Our approach is applied to two real objects, and experimental results are shown in Section 4. Concluding remarks are presented in Section 5.

\section{Measuring Shape and Reflectance Properties}

This section describes a method to recover object surface reflectance properties from a range image and a color image sequence. First, we describe the image acquisition system in our analysis followed by a description of the algorithm used for reflectance parameter estimation.

\subsection{Image Acquisition System}

A range image is obtained using a light-stripe range finder with a liquid crystal shutter and a color CCD video camera [8]. It can compute 3D point locations corresponding to image pixels based on optical triangulation. For each range image, a set of three images is obtained containing $X, Y$ and $Z$ locations of all pixels. Each of the range-image pixels represents an $(X, Y, Z)$ location of a corresponding point on an object surface. The same color camera is used for digitizing color images. Therefore, pixels of the range images and the color images directly correspond. The color video camera is calibrated to ensure linear response from all three color bands. The experimental setup used in our experiments is illustrated in Fig. 1. A 


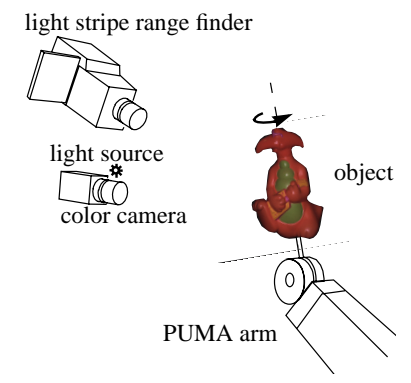

Figure 1: Image acquisition system

single incandescent lamp is used as a point light source. In our experiments, the light source color is assumed to be given by calibration. The light source is located nearby the camera lens so that both the viewing direction and the illumination direction are approximately the same. This light source location is chosen to avoid the problem of self-shadowing.

First, the range finder is calibrated using a calibration box of known size and shape. The calibration produces a $3 \times 4$ matrix $\Pi$ which represents the projection transformation between the world coordinate system and the image coordinate system. The object whose shape and reflectance information are to be recovered is mounted to the end of a PUMA 560 manipulator whose location with respect to the world coordinate system is given by calibration. The object orientation is controlled using the PUMA manipulator, and the object location is given as a $4 \times 4$ transformation matrix $T$ for each digitized image. First, one range image is captured to determining the shape of the object surface facing to the camera. Then, a sequence of color images is captured at a fixed angle step of object orientation. The projection transformation matrix $\Pi$ and the transformation matrix $T$ will be used for determining an observed color sequence for each of the range image pixels. The observed color sequence represents how a surface point corresponding to the range image pixel appears as the object rotates, and it will be used for analyzing reflectance properties of the object.

\subsection{Observed Color Sequence for Each Point on Object Surface}

For measuring reflectance properties of the object surface, a sequence of observed colors has to be determined for each point on the object surface. In this section, we describe how to determine the observed color sequence.

We represent world coordinates and image coordinates using homogeneous coordinates. A point on the object surface with Euclidean coordinates $(X, Y, Z)$ is expressed by a column vector $P=[X, Y, Z, 1]^{T}$. An image pixel location $(x, y)$ is represented by $p=[x, y, 1]^{T}$. As described in the previous section, the camera projection transformation is represented by a $3 \times 4$ matrix $\Pi$, and the object location and orientation are given by a $4 \times 4$ object transformation matrix $T$. We represent 
the object transformation matrix for the frontal range image by $T_{\text {range, }}$ and that for the input color image frame $f$ by $T_{f}(f=1 \ldots n)$. Thus, the projection of a range image pixel (i.e., corresponding point on the object surface) in the color image frame $f$ is given as

$$
p_{f}=\prod T_{f} T_{\text {range }}^{-1} P(f=1 \ldots n)
$$

where the last component of $p_{f}$ has to be normalized to give the projected image location $(x, y)$. The observed color of the range-image pixel in the color image frame $f$ is given as the $(R, G, B)$ color intensity at the pixel location $(x, y)$. To avoid unnecessary aliasing, bilinear interpolation is used to sample the color intensity. If the range image pixel is not visible in the color image (i.e., the surface point is facing away from the camera, or it is occluded), the observed color for the pixel is set to $(R, G, B)=(0,0,0)$. By repeating this for all object orientations, we finally get an observed color sequence for each of the range image pixels.

\subsection{Reflection Model}

In this section, we describe the reflectance model used in this work. The reflectance model will be used later for separating the diffuse and surface reflection components from a sequence of color images. The reflection component separation is described in Section 2.4. The model will also be used for reflectance parameter estimation which is explained in Section 2.5.

A general reflection model is described in terms of three reflection components, namely the diffuse lobe, the specular lobe, and the specular spike [7]. Unlike the diffuse lobe and the specular lobe components, the specular spike component is not commonly observed in many actual applications. The component can be observed only from mirror-like smooth surfaces where reflected light rays of the specular spike component are concentrated in a specular direction. It is, thus, difficult to observe the specular spike component from a coarsely sampled set of viewing directions. Therefore, in many computer vision and computer graphics applications, reflection models are represented by linear combinations of two reflection components: the diffuse lobe component and the specular lobe component. These two reflection components are normally called the diffuse reflection component and the specular reflection component, respectively. This reflection model was formally introduced by Shafer as the dichromatic reflection model [12]. The Torrance-Sparrow model [13] is used in our analysis for representing the diffuse and specular reflection components. As Fig. 1 illustrates, the illumination and viewing directions are fixed and are assumed to be the same. The reflection model used for the particular experiment setup is given as

$$
I_{m}=K_{D, m} \cos \theta+K_{S, m} \frac{1}{\cos \theta} \exp \frac{-\theta^{2}}{2 \sigma_{\alpha}^{2}} \quad m=R, G, B
$$

where $\theta$ is the angle between the surface normal and the viewing direction (or the light source direction), $K_{D, m}$ and $K_{S, m}$ are constants for the respective reflection 
components, and $\sigma_{\alpha}$ is the standard deviation of a facet slope $\alpha$ of the TorranceSparrow model. For the derivation of the reflection model, see the appendices of [10]. The direction of the light source and the camera with respect to the surface normal is referred as the sensor direction $\theta$ in this paper. In our analysis, only reflections which bounce only once from the light source are considered. Therefore, the reflection model is valid only for convex objects, and it cannot represent interreflections on concave object surfaces. We empirically found that interreflection did not affect our analysis significantly.

\subsection{Reflection Component Separation}

The algorithm to separate the two reflection components is described here. The separation of the diffuse and specular components is important for robust estimation of reflectance parameters. It has been reported that estimating all reflectance parameters at once tends to make computation unstable [4]. Therefore, the separation algorithm is applied prior to reflectance parameter estimation. The separation algorithm was originally introduced for the case of a moving light source by Sato and Ikeuchi [9]. In this paper, a similar algorithm is applied for the case of a moving object.

Using three color bands; red, green, and blue, the coefficients $K_{D, m}$ and $K_{S, m}$, in Eq.(2), becomes two linearly independent vectors, $\vec{K}_{D}$ and $\vec{K}_{S}$, unless the colors of the two reflection components are accidentally the same

$$
\vec{K}_{D}=\left[\begin{array}{lll}
K_{D, R} & K_{D, G} & K_{D, B}
\end{array}\right]^{T} \quad \vec{K}_{S}=\left[\begin{array}{lll}
K_{S, R} & K_{S, G} & K_{S, B}
\end{array}\right]^{T}
$$

These two vectors represent the colors of the diffuse and specular reflection components in the dichromatic reflectance model [12].

First, the color intensities in the R, G, and B channels from $n$ input images of the object are measured for each range image pixel as described in Section 2.2. It is important to note that all intensities are measured for the same range image pixel. The three sequences of intensity values are stored in the columns of an $n \times 3$ matrix $M$. Considering the reflectance model and two color vectors in Eq.(2) and Eq.(3), the intensity values in the $\mathrm{R}, \mathrm{G}$, and $\mathrm{B}$ channels can be represented as

$$
\begin{aligned}
M & =\left[\begin{array}{lll}
\vec{M}_{R} & \vec{M}_{G} & \vec{M}_{B}
\end{array}\right] \\
& =\left[\begin{array}{cc}
\cos \theta_{1} & E\left(\theta_{1}\right) \\
\cos \theta_{2} & E\left(\theta_{2}\right) \\
\vdots & \vdots \\
\cos \theta_{n} & E\left(\theta_{n}\right)
\end{array}\right]\left[\begin{array}{ccc}
K_{D, R} & K_{D, G} & K_{D, B} \\
K_{S, R} & K_{S, G} & K_{S, B}
\end{array}\right] \\
& =\left[\begin{array}{ll}
\vec{G}_{D} & \vec{G}_{S}
\end{array}\right]\left[\begin{array}{l}
\vec{K}_{D}^{T} \\
\vec{K}_{S}^{T}
\end{array}\right] \\
& \equiv G K
\end{aligned}
$$


where $E(\theta)=\left(\exp \left(-\theta^{2} / 2 \sigma_{\alpha}{ }^{2}\right)\right) / \cos \theta$, the two vectors $\overrightarrow{G_{D}}$ and $\vec{G}_{S}$ represent the intensity values of the diffuse and specular reflection components with respect to the sensor direction $\theta$. The vectors $\vec{K}_{D}$ and $\vec{K}_{S}$ represent the diffuse and the specular reflection color vectors, respectively. We call the two matrices $G$ and $K$, the geometry matrix and the color matrix, respectively.

Suppose we have an estimate of the color matrix $K$. Then, the two reflection components represented by the geometry matrix $G$ are obtained by projecting the observed reflection stored in $M$ onto the two color vectors $\vec{K}_{D}$ and $\vec{K}_{S}$.

$$
G=M K^{+}
$$

where $K^{+}$is the $3 \times 2$ pseudoinverse matrix of the color matrix $K$.

The derivation shown above is based on the assumption that the color matrix $K$ is known. In our experiments, the specular reflection color vector $\vec{K}_{S}$ is directly measured by a calibration procedure. Therefore, only the diffuse color vector $\vec{K}_{D}$ is unknown and needs to be determined.

From Eq.(2), it can be seen that the distribution of the specular reflection component is limited to a fixed angle, depending on $\sigma_{\alpha}$. Therefore, if two vectors, $\vec{w}_{i}=\left[I_{R i} I_{G i} I_{B i}\right]^{T}(i=1,2)$ are sampled on the $\theta$ axis at large enough interval, at least one of these vectors will be equal to the color vector of the diffuse reflection component $\vec{K}_{D}$. (i.e., it has no specular reflection component.) The desired color vector of the diffuse reflection component $\vec{K}_{D}$ is the vector $\vec{w}_{i}$ which subtends the largest angle with respect to the vector $\vec{K}_{S}$. The angle between the two color vectors can be calculated as $\beta=\arccos \left(\vec{K}_{S} \cdot \vec{w}_{i} /\left(\left|\vec{K}_{S}\right|\left|\vec{w}_{i}\right|\right)\right)$.

Once we get the color matrix $K$, the geometry matrix $G$ can be calculated from Eq.(5). Each of the diffuse and specular reflection components is then given as

$$
M_{D}={\overrightarrow{G_{D}}}_{\vec{K}_{D}}^{T} \quad M_{S}={\overrightarrow{G_{S}}}_{\hat{K}_{S}}{ }^{T}
$$

\subsection{Specular Parameter Estimation for Segmented Regions}

In the previous section, we described our method for separating the two reflection components from a sequence of observed colors of each range image pixel. In this section, we will discuss how to estimate parameters of the reflectance model for the range image pixel using the separated reflection components.

By applying the separation algorithm as explained above, we obtain a sequence of the diffuse reflection intensities and a sequence of the specular reflection intensities for each range image pixel. This information is sufficient to estimate reflectance parameters of the reflection model Eq.(2) separately for the two reflection components. As Eq.(2) shows, the reflectance model is a function of the angle between the surface normal and the viewing direction $\theta$. Therefore, for estimating reflectance parameters: $K_{D, m}, K_{S, m}$, and $\sigma_{\alpha}$, the angle $\theta$ has to be computed as the object is rotated. Since the projection transformation matrix is already given and the object orientation is known in the world coordinate system, it is straightforward 
to compute the surface normal vector and a viewing direction vector (or a illumination vector) at a surface point corresponding to each range image pixel. Thus, the angle $\theta$ between the surface normal and the viewing direction vector can be computed. After the angle $\theta$ is computed, the reflectance parameters for the diffuse reflection component $\left(K_{D, m}\right)$ and the specular reflection component $\left(K_{S, m}\right.$ and $\left.\sigma_{\alpha}\right)$ are estimated separately. In our experiments, the camera output is calibrated so that the specular reflection color has the same value from the three color channels. Therefore, only one color band is used to estimate $K_{S}$ in our experiment.

By repeating the estimation procedure for all range image pixels, we can estimate the diffuse reflection component parameters for all range image pixels if those pixels are illuminated in one or more frames of the image sequence. On the other hand, the specular reflection component can be observed only from a few viewing directions. Due to this fact, the specular reflection component can be observed only in a small subset of all range image pixels. We cannot estimate the specular reflection component parameters for those pixels in which the specular reflection component is not observed. Even if the specular reflection component is observed, the parameter estimation can become unreliable if the specular reflection is not sufficiently strong.

For the above reasons, we decided to assign the specular reflection component parameters based on region segmentation. In our experiments, it is assumed that the object surface can be segmented into a finite number of regions which have uniform diffuse color, and all range image pixels within each region have the same specular reflection component parameters. By using the segmentation algorithm, the specular reflection parameters of each region can be estimated from range image pixels with strong specularity. The estimated parameters are assigned to the rest of range image pixels in the region. The range image pixels with strong specularity can be easily selected after the reflectance components are separated. The limitation of this approach is that the specular reflection parameters for a region cannot be estimated if no specularity is observed in the region. In that case, the specular reflection parameters of neighboring regions can be assigned to the region as an approximation. It is important to note that segmentation and parameter estimation are used only for the specular reflection component. The diffuse reflection component parameters are estimated locally regardless of specularity.

After reflectance parameters are estimated for each of the range image pixels, each range image pixel has 5 associated reflectance parameters $\left(K_{D, R}, K_{D, G}, K_{D, B}\right.$, $K_{S}$, and $\left.\sigma_{\alpha}\right)$. Combining those parameters with the $(X, Y, Z)$ location of the range image pixel, we obtain a new image with 8 bands $\left(X, Y, Z, K_{D, R}, K_{D, G}, K_{D, B}\right.$, $K_{S}$, and $\left.\sigma_{\alpha}\right)$. We call this image an object image since it contains both shape and reflectance properties of the object. Using this technique, we obtain the object images of two real objects. These object images will be later used for creating a new object model whose shape and reflectance properties are interpolated from the two real objects. 


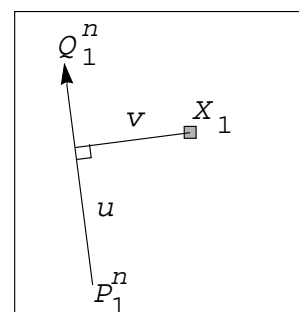

the first object image

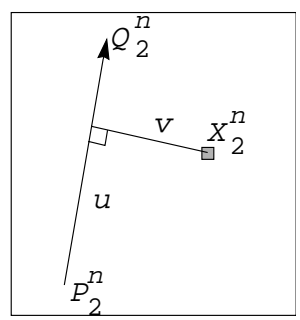

the second object image

Figure 2: Pixel correspondence by a line pair

\section{Interpolation of Shape and Reflectance Properties}

After object images of the real objects are created, we can generate new object images by interpolating between the obtained object images. For this purpose, we need to define pixelwise correspondence between the two object images. In this section, we describe how to define the pixelwise correspondence and how to interpolate the two object images based on the correspondence.

Several algorithms for establishing pixelwise correspondence between two images have been developed. In our analysis, we used the mapping introduced by Beier and Neely [1] to define the pixelwise correspondence between the two object images, each of which has 8 bands $\left(X, Y, Z, K_{D, R}, K_{D, G}, K_{D, B}, K_{S}\right.$, and $\left.\sigma_{\alpha}\right)$. A user selects $m$ pairs of corresponding line features each of which is specified by pairs of pixel coordinates $P_{i}^{n} Q_{i}^{n}$ where $i$ is the image number $(i=1,2)$ and $n$ is the index of the line pair $(n=1 \ldots m)$. First, we consider a mapping defined by one pair of lines in the two object images (Fig. 2). A pair of corresponding lines $P_{1}^{n} Q_{1}^{n}$ in the first object image and $P_{2}^{n} Q_{2}^{n}$ in the second object image defines a coordinate mapping from the first image pixel coordinate $X_{1}$ to the second image pixel coordinate $X_{2}^{n}$ such that

$$
\begin{gathered}
u=\frac{\left(X_{1}-P_{1}^{n}\right) \cdot\left(Q_{1}^{n}-P_{1}^{n}\right)}{\left\|Q_{1}^{n}-P_{1}^{n}\right\|^{2}} \quad v=\frac{\left(X_{1}-P_{1}^{n}\right) \cdot\left(Q_{1}^{n}-P_{1}^{n}\right)_{\perp}}{\left\|Q_{1}^{n}-P_{1}^{n}\right\|} \\
X_{2}^{n}=P_{2}^{n}+u\left(Q_{2}^{n}-P_{2}^{n}\right)+\frac{v\left(Q_{2}^{n}-P_{2}^{n}\right)_{\perp}}{\left\|Q_{2}^{n}-P_{2}^{n}\right\|}
\end{gathered}
$$

where $(V)_{\perp}$ is the vector perpendicular to and the same length as the input vector $V$. (There are two perpendicular vectors defined this manner. Either the left or right one can be used as long as it is consistently used throughout.) The value $u$ represents the position along the line segment $P_{i}^{n} Q_{i}^{n}$. The value $u$ is 0 at the point $P_{i}^{n}$ and 1 at the point $Q_{i}^{n}$, and can be less than 0 and greater than 1 outside the line segment $P_{i}^{n} Q_{i}^{n}$. The value $v$ represents the perpendicular distance from $X$ to the line segment $P_{i}^{n} Q_{i}^{n}$. Then, Eq.(8) determines the second image coordinate $X_{2}^{n}$ which corresponds to the first image coordinate $X_{1}$ based on the $n$th line pairs $P_{1}^{n} Q_{1}^{n}$ and $P_{2}^{n} Q_{2}^{n}(n=1 \ldots m)$. 
For multiple pairs of lines, the second image coordinate $X_{2}$ is given by a linear combination of displacements $X_{2}^{n}-X_{1}$ for all line pairs $(n=1 \ldots m)$. The weight of the linear combination is determined by the distance from the first image coordinate $X_{1}$ to the line segment $P_{1}^{n} Q_{1}^{n}$ as

$$
w_{n}=\left\{\frac{\left\|Q_{1}^{n}-P_{1}^{n}\right\|^{p}}{a+\operatorname{dist}\left(X_{1}, P_{1}^{n} Q_{1}^{n}\right)}\right\}^{b}
$$

where $a, b$ and $p$ are constants which are used to change the relative effect of the lines. In our implementation, we used $a=0.1, b=2.0$, and $p=0.2$.

Finally, the second image coordinate $X_{2}$ is given as

$$
X_{2}=X_{1}+\frac{\sum_{n} w_{n}\left(X_{2}^{n}-X_{1}\right)}{\sum_{n} w_{n}} .
$$

Once the pixelwise correspondences are established, an intermediate object image can be created as linear interpolation of the two object images. For each pixel $X_{1}$ of the first object image, the corresponding pixel $X_{2}$ in the second object image is determined. Then, a new intermediate object image pixel is given as linear interpolation of values of the two object image pixels $X_{1}$ and $X_{2}$. Smooth transition of object shapes and reflectance properties are obtained by gradually changing the ratio of the interpolation.

\section{Experimental Results}

\subsection{Measurement}

In the previous sections, we described our method for obtaining new object models whose shape and reflectance properties are interpolated from two real objects. The method includes four steps: (1) separate the two fundamental reflection components using a range image and a sequence of color images, (2) estimate the reflection model parameters from the separated reflection components, and store them with the $(X, Y, Z)$ coordinates as an object image, (3) establish pixelwise correspondences between two object images, and create an intermediate object image by interpolating the two object images, and (4) synthesize images of the interpolated object using Eq.(2). We applied the method to actual range and color images taken in a laboratory setup in order to demonstrate the feasibility of the proposed method.

The target objects used in our experiment are two plastic toys with an approximate height of $170 \mathrm{~mm}$. These objects are multi-colored, and each painted surface region appears to have a uniform color. One frontal range image and a sequence of color images of each object are taken using the image acquisition system described in Section 2.1. A frontal range image is captured before rotating the object (object orientation $=0^{\circ}$ ). Then, a sequence of color images are taken by rotating the object. In our experiment, color images are taken at $3^{\circ}$ intervals from $-90^{\circ}$ to $90^{\circ}$ 
( 60 color images total). The input range images for the two plastic toys are shown as a mesh in Fig. 3. The input color image sequences of the objects are shown in Fig. 4 and Fig. 5 .

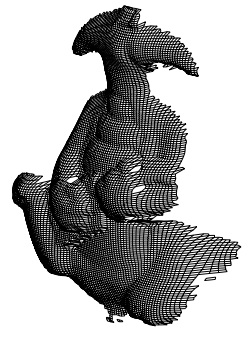

object 1

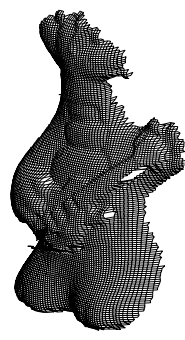

object 2

Figure 3: Measured shapes

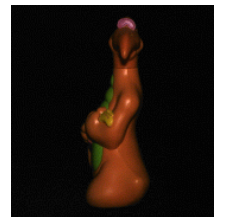

frame 0

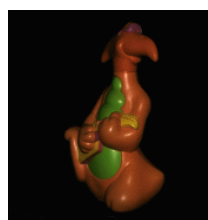

frame 15

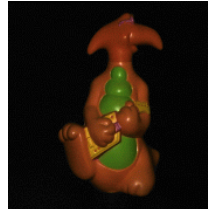

frame 30

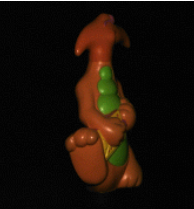

frame 45

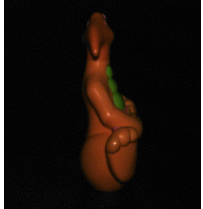

frame 59

Figure 4: Input color sequence for object 1

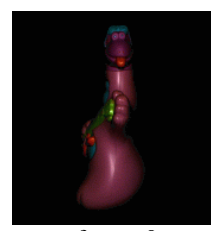

frame 0

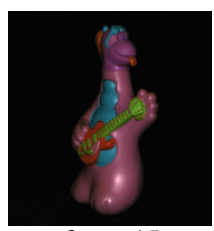

frame 15

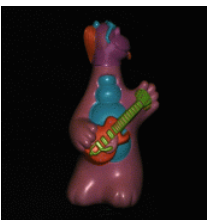

frame 30

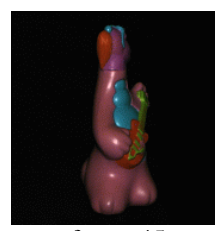

frame 45

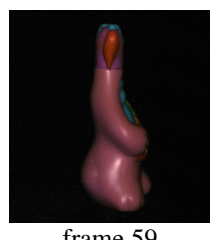

frame 59

Figure 5: Input color sequence for object 2

\subsection{Reflection Component Separation}

By projecting a range image pixel onto all input color images, a sequence of observed colors is determined for the range image pixel as explained in Section 2.2. Fig. 6 (a) illustrates a typical observed color sequence for a range image pixel with specularity. The intensities are set to zero before image frame 8 and after image frame 53 because the surface point corresponding to the range image pixel is not visible from the camera due to self-occlusion. The specular reflection component can be observed near image frame 29. When the specular reflection component exists, the 
output color intensity is a linear combination of the diffuse reflection component and the specular reflection component. In this example, the observed specular reflection is relatively small compared to the diffuse reflection component. As a result, estimating reflectance parameters for both the diffuse and specular reflection components together could be sensitive to various disturbances such as image noise. That is why the reflection component separation is performed in prior to parameter estimation in our analysis. By separating the two reflection components, reflectance parameters can be estimated separately in a robust manner.

The algorithm to separate the diffuse and specular reflection components was applied to the observed color sequence for each range image pixel. The red, green, and blue intensities of the observed color sequence are stored in the matrix $M$ as its columns Eq.(4). Then, the matrix $G$ is computed from the matrix $M$ and the matrix $K$ which is estimated as described in Section 2.4. Finally, the diffuse and specular reflection components are given as shown in Eq.(6). This reflection component separation is repeated for all range image pixels. The separation result for the observed color change in Fig. 6 (a) is shown in Fig. 6 (b). After the reflection component separation, reflectance parameters can be estimated separately. The result of parameter estimation will be shown in Section 4.3.
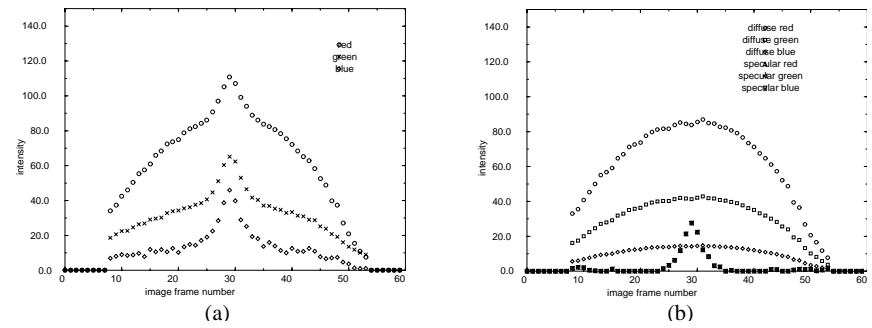

Figure 6: Observed color sequence (a) and separation result (b)

\subsection{Reflectance Parameter Estimation for Segmented Regions}

By using the separated diffuse reflection components, the object surface was segmented based on the hue of the diffuse reflection components, as explained in Section 2.5. The results of the region segmentation are shown in Fig. 7 (segmented regions are represented as grey levels). For estimating specular reflection component parameters, 10 range image pixels with the largest specular reflection component are selected for each of the segmented regions. Then, the specular reflection component parameters of the reflection model Eq.(2) are estimated for each of those selected range image pixels. Finally, the average of the estimated parameters of the selected range image pixels is used as the specular reflection component parameters of the segmented region. The estimated specular reflection parameters are assigned to all range image pixels within the segmented region. The results of the estimated specular reflection component parameters are shown in Table 1 and Table 2. 


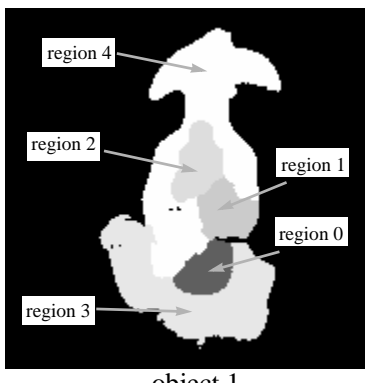

obiect 1

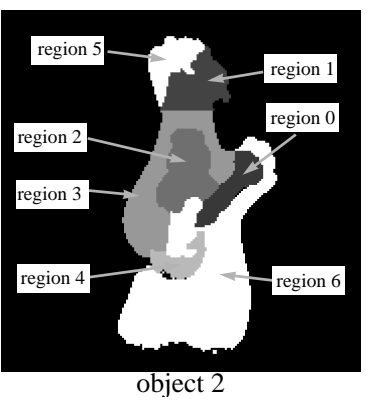

object 2

Figure 7: Segmented object surface

Unlike the specular reflection parameter estimation, parameters of the diffuse reflection component $\left(K_{D, R}, K_{D, G}, K_{D, B}\right)$ are estimated for each range image pixel individually. That is because the diffuse reflection component at the range image pixel is guaranteed to be observed as long as the range image pixel is illuminated and not occluded from the viewing direction. The results of the diffuse reflection component parameter estimation are shown in Fig. 8. which shows the estimated parameters $K_{D, R}, K_{D, G}$, and $K_{D, B}$ as a color image.

Table 1. Estimated specular parameters for the object 1

\begin{tabular}{cc|cc}
\hline & & $K_{s}$ & $\sigma_{\alpha}$ \\
\hline \multirow{4}{*}{ region } & 0 & 17.31 & 0.301 \\
& 1 & 37.00 & 0.280 \\
& 2 & 40.33 & 0.269 \\
& 3 & 42.17 & 0.301 \\
& 4 & 46.38 & 0.238 \\
\hline
\end{tabular}

Table 2. Estimated specular parameters for the object 2

\begin{tabular}{cc|cc}
\hline & & $K_{s}$ & $\sigma_{\alpha}$ \\
\hline \multirow{4}{*}{ region } & 0 & 36.82 & 0.293 \\
& 1 & 43.69 & 0.187 \\
& 2 & 78.15 & 0.166 \\
& 3 & 78.55 & 0.197 \\
& 4 & 82.71 & 0.231 \\
& 5 & 91.54 & 0.166 \\
& 6 & 43.69 & 0.189 \\
\hline
\end{tabular}

By combining the five estimated parameters $\left(K_{D, R}, K_{D, G}, K_{D, B}, K_{S}\right.$, and $\left.\sigma_{\alpha}\right)$ and three measured values $(X, Y, Z)$, we obtain object images for the two real objects. These two object images are used to create intermediate object images which have interpolated shape and reflectance properties. 


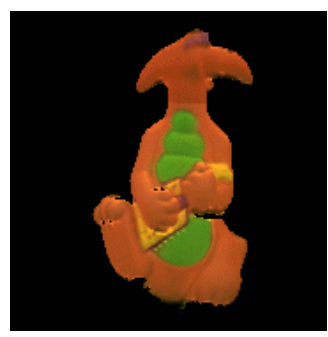

object 1

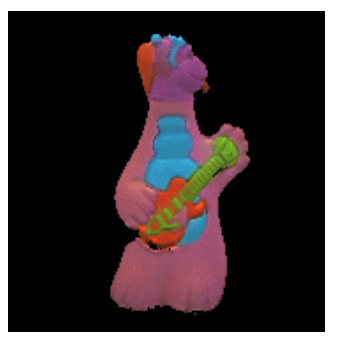

object 2

Figure 8: Estimated $K_{D}$
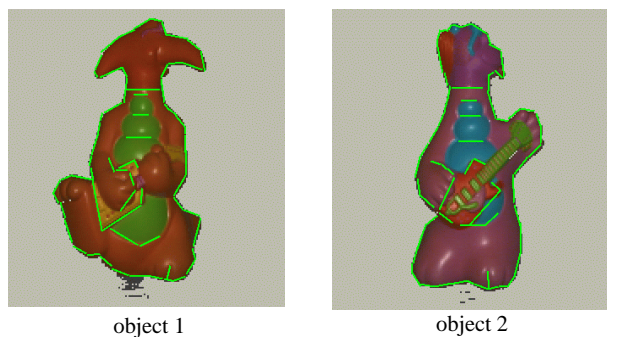

Figure 9: Edited line pairs (70 line pairs are shown in green)

\subsection{Interpolation of Shape and Reflectance Parameters}

By using the two object images, an interpolated object shape and reflectance properties are created as explained in Section 3. Fig. 9 shows 70 manually drawn corresponding line pairs on the two object images in green. Based on the line pairs, pixelwise correspondence between the two object images is determined. Then, the two images are linearly interpolated to produce an intermediate object image. An example of the interpolated object shape and reflectance parameters are shown in Fig. 10. In this particular example, $50 \%-50 \%$ interpolation was used.
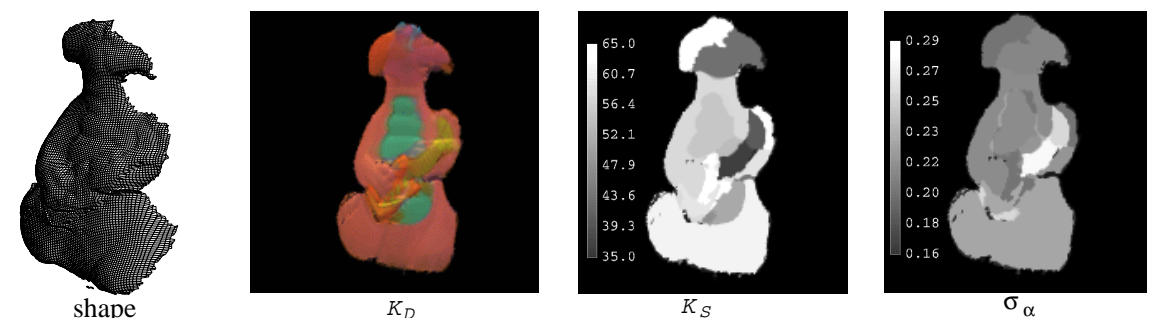

Figure 10: Interpolation of shape, $K_{D}, K_{S}$, and $\sigma_{\alpha}$ 


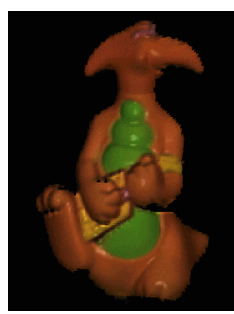

$100 \%-0 \%$

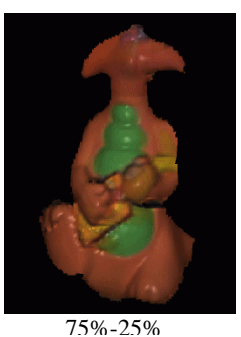

$75 \%-25 \%$

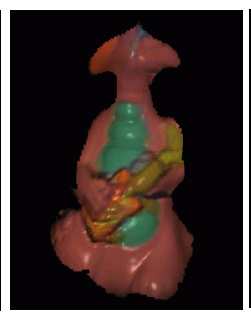

$50 \%-50 \%$

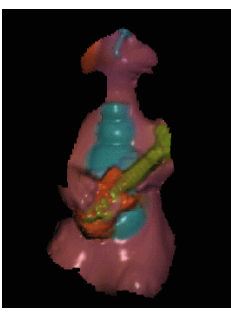

$25 \%-75 \%$

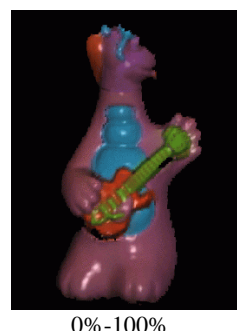

$0 \%-100 \%$

Figure 11: Synthesized interpolated objects

\subsection{Morphing Results}

Finally, the interpolated object shape and reflectance properties are used to create new color images in the morph. Fig. 11 shows a synthesized color image of the object for $50 \%-50 \%$ interpolation along with synthesized images for other interpolation ratios which are created in the same manner as 50\%-50\% interpolation. The same viewing direction, light source direction, and object orientation are used for rendering Fig. 11 as for frame 30 of the input color images in Fig. 4 and Fig. 5. It is important to see that specularity appears naturally in those synthesized color images. This is because, unlike other methods, our method uses intermediate reflectance parameters, as well as intermediate shapes, which are computed separately from the two real objects. Therefore, images rendered using our approach do not contain false specularities which are often created by other $2 \mathrm{D}$ or $3 \mathrm{D}$ morphing techniques.

\section{Conclusion}

We have studied an approach for 3D shape and reflectance property morphing of two real objects. Unlike previously developed 3D shape morphing techniques, our approach uses object surface reflectance properties as well as object shapes for creating intermediate morphing results. This enables us to render images with correct shading such as highlights and shadows. Our morphing method consists of two components: shape and reflectance property measurement, and smooth interpolation of those measured properties. First, a range image and a color image sequence are used for obtaining an object shape and reflectance properties. The estimation of the reflection parameters is performed reliably by separating the reflection components. The obtained shape and reflectance parameters are stored in an extended range image which we call object image. The reflection model used in our analysis is described as a linear combination of the Lambertian model and a simplified Torrance-Sparrow model. After object images are created for the two real objects, the object images are used to create a new intermediate object image which has interpolated shape and reflectance properties. Finally, the newly created intermediate object image is used to render new images of the objects in the morph. The proposed approach has been applied to real range and color images of two plas- 
tic objects, and the effectiveness of the proposed approach has been successfully demonstrated by showing intermediate morphing results with proper shadings.

\section{References}

1. T. Beier and S. Neely, Feature-based image matamorphosis, Computer Graphics, vol. 26, no. 2, (July 1992) pp. 35-42. Proceedings of SIGGRAPH 92.

2. T. He, S. Wang, and A. Kaufman, Wavelet-based volume morphing, in Proceedings of IEEE/ACM SIGGRAPH Visualization '94, Los Alamitos, CA (October 1994) pp. $85-91$.

3. K. Ikeuchi and K. Sato, Determining reflectance properties of an object using range and brightness images, IEEE Transactions on Pattern Analysis and Machine Intelligence, vol. 13, no. 11 (November 1991) pp. 1139-1153.

4. G. Kay and T. Caelli, Inverting an illumination model from range and intensity maps, CVGIP: Image Understanding, vol. 59, no. 2 (March 1994) pp. 183-201.

5. J. R. Kent, W. E. Carlson, and R. E. Parent, Shape transformation for polyhedral objects, Computer Graphics, vol. 26, no. 2, New York, NY (July 1992) pp. 47-54. Proceedings of SIGGRAPH 92.

6. A. Lerios, C. D. Garfinkle, and M. Levoy, Feature-based volume metamorphosis, Computer Graphics, Los Angeles, CA (August 1995) pp. 449-456. Proceedings of SIGGRAPH 95.

7. S. Nayar, K. Ikeuchi, and T. Kanade, Surface reflection: physical and geometrical perspectives, IEEE Transactions on Pattern Analysis and Machine Intelligence, vol. 13, no. 7, (1991) pp. 611-634.

8. K. Sato, H. Yamamoto, and S. Inokuchi, Range imaging system utilizing nematic liquid crystal mask, in Proceedings of International Conference on Computer Vision, (1987) pp. 657-661.

9. Y. Sato and K. Ikeuchi, Temporal-color space analysis of reflection, Journal of Optical Society of America A, vol. 11, no. 11 (November 1994) pp. 2990-3002.

10. Y. Sato and K. Ikeuchi, Reflectance analysis for 3D computer graphics model generation, Graphical Models and Image Processing, vol. 58, no. 5 (September 1996) pp. 437-451.

11. T. W. Sederberg, P. Gao, G. Wang, and H. Mu, 2-D shape blending: An intrinsic solution to the vertex path problem, in Proceedings of $A C M S I G G R A P H$ 93, New York, NY (August 1993) pp. 15-18.

12. S. Shafer, Using color to separate reflection components, COLOR Research, vol. 10, no. 4, (1985) pp. 210-218.

13. K. E. Torrance and E. M. Sparrow, Theory for off-specular reflection from roughened surface, Journal of Optical Society of America, vol. 57 (1967) pp. 1105-1114. 